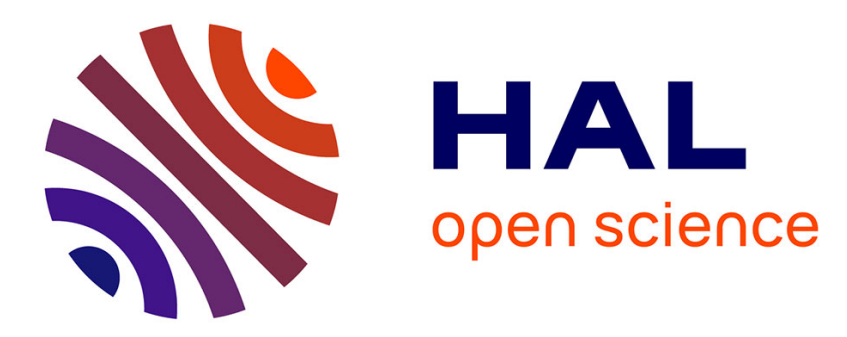

\title{
Agir en faveur de la qualité de vie au travail : présentation d'une recherche-action innovante étudiant les interactions entre acteurs internes et processus d'intervention
}

N. Guidou, P. Sarnin

\section{To cite this version:}

N. Guidou, P. Sarnin. Agir en faveur de la qualité de vie au travail : présentation d'une recherche-action innovante étudiant les interactions entre acteurs internes et processus d'intervention. Psychologie du travail et des organisations, 2019, 25, pp.75 - 85. 10.1016/j.pto.2019.01.001 . hal-03480677

\section{HAL Id: hal-03480677 \\ https://hal.science/hal-03480677}

Submitted on 20 Dec 2021

HAL is a multi-disciplinary open access archive for the deposit and dissemination of scientific research documents, whether they are published or not. The documents may come from teaching and research institutions in France or abroad, or from public or private research centers.
L'archive ouverte pluridisciplinaire HAL, est destinée au dépôt et à la diffusion de documents scientifiques de niveau recherche, publiés ou non, émanant des établissements d'enseignement et de recherche français ou étrangers, des laboratoires publics ou privés.

\section{다(1) $\$$}

Distributed under a Creative Commons Attribution - NonCommerciall 4.0 International 
Agir en faveur de la qualité de vie au travail : présentation d'une recherche-action innovante étudiant les interactions entre acteurs internes et processus d'intervention

\section{Developing quality of working life: presentation of an innovative research-action investigating interactions between internal actors and process intervention}

Nadège GUIDOU,

Université Lumière Lyon 2, Laboratoire GREPS, Institut de Psychologie

5 avenue Pierre Mendes France, 69576 Bron cedex

nadege.guidou@univ-lyon2.fr

\section{Philippe SARNIN}

Université Lumière Lyon 2, Laboratoire GREPS, Institut de Psychologie

5 avenue Pierre Mendes France, 69576 Bron cedex, philippe.sarnin@univ-lyon2.fr 
Agir en faveur de la qualité de vie au travail : présentation d'une recherche-action innovante étudiant les interactions entre acteurs internes et processus d'intervention

Developing quality of working life: presentation of an innovative research-action investigating interactions between internal actors and process intervention 


\title{
Résumé :
}

Cet article présente une recherche-action étudiant les interactions entre acteurs internes et processus d'intervention lors d'une démarche de santé au travail. Le travail de la demande a abouti un cadre méthodologique inédit, permettant à un collectif d'acteurs internes de s'impliquer activement. Cette haute implication s'est traduite par leur co-construction et leur co-réalisation d'une enquête de santé à grande échelle. Nous montrons comment cette expérience a permis de développer leurs connaissances sur la dynamique santé-travail mais aussi de passer d'une enquête relevant les impacts négatifs du travail sur la santé, à une démarche de qualité de vie au travail. Nous analysons également les difficultés importantes rencontrées par les acteurs particulièrement lors du passage entre le diagnostic et les transformations.

\begin{abstract}
:
This paper presents a research-action investigating interactions between internal actors and process intervention during an approach of health at work. The work of the request was culminated in a novel methodology allows a group of internal actors to become actively involved. This high level of involvement has resulted in co-construction and co-production of a large-scale health survey. We show how this experience has enabled them to develop their knowledge of the interactions between health and work but also to move from an approach that identified the health impacts of work to a quality of working life approach. Moreover, we analyse important difficulties encountered by actors especially during the transition from diagnosis to transformation.
\end{abstract}

\section{Mot-clés :}

Intervention, transformation, qualité de vie au travail, engagement des acteurs, interaction

\section{Key-words :}

Intervention, transformation, quality of working life, involvement of actors, interaction 
Cet article rend compte d'une recherche-action questionnant les interactions existant entre les acteurs internes d'un milieu de travail et le processus à l'œuvre au cours d'une intervention en santé au travail. Après en avoir expliqué l'intérêt, nous détaillerons la méthodologie inédite élaborée pour permettre à un collectif d'acteurs internes, de construite et de conduire euxmêmes une démarche entourant les liens entre santé et travail. Ancrés dans le cadre épistémologique de la recherche fondamentale de terrain, nous présenterons des résultats éclairant les effets de cette haute implication des acteurs internes notamment au regard des freins auxquels ils ont été exposés. Nous en déduirons de nouvelles perspectives de recherche.

\section{Des liens entre santé et travail à l'interaction entre acteurs et processus d'intervention}

Dès les années 1990, les médias français se sont emparés des liens entre travail et santé en relayant des drames humains (Ponnelle, Vaxevanoglou, 2017). Cette manière d'appréhender ces liens est également celle de la loi : l'obligation de résultat des employeurs français, vise la suppression des effets pathogènes du travail sur la santé mais n'aborde pas ses aspects vertueux. Au niveau de la recherche et de la pratique aussi, les travaux ont assez longtemps conservé cette perspective : les modèles causalistes du stress (Karasek, 1979 ; Siegrist, 2001), interactionnistes (Lassare, 2005), transactionnels (Lazarus \& Folkman, 1984) puis le modèle multifactoriel des RPS (Askenazy et coll., 2010) partagent un fondement positiviste et épidémiologique (Althaus, 2013) : l'intervention y est centrée sur un relevé des altérations causées par le travail sur la santé. Ces approches considèrent alors que les salariés sont passifs devant les difficultés et conçoivent la santé non comme un processus en évolution, mais comme un état normal (Clot, 2010) qu'il faudrait protéger (Vezina et coll., 2015). De plus, en déléguant la question des RPS aux experts du fait de leur complexité (Boussard, 2009), ces démarches n'ouvrent pas d'espaces de discussion autour des conditions concrètes du travail (Clot et Davezies, 2011 ; Rouat, 2010). Les méthodologies ont donc évolué vers des approches combinant méthodes quantitatives et qualitatives (Fielding \& Schreier, 2001) et incluant plus de participation des acteurs de l'entreprise (Jeoffrion et coll., 2014). Pour autant, les débats restent majoritairement centrés sur les altérations. Ces premiers travaux législatifs, réglementaires, scientifiques ou pratiques sont donc globalement passés à côté de la réinterrogation pourtant ouverte par la question des RPS autour du travail conçu «comme un processus de développement social, identitaire, comme lieu d'échanges et de solidarité qui participe à fonder une société » (Valléry et Leduc, 2012, p39).

De nouvelles perspectives s'ouvrent en France en 2010 avec le rapport Lachmann et coll. puis en 2013 par la signature de l'accord pour le développement de la qualité de vie au travail (QVT). Visant l'articulation entre performances économique et sociale (Caillé et Jeoffrion, 2017), les démarches QVT tentent de (re)construire une «philosophie d'entreprise » (Grosjean et Guyot, 2016) en engageant les acteurs patronaux, salariaux et syndicaux à débattre pour dessiner un avenir commun (Levet, 2013). Si ces orientations permettent effectivement de revenir aux fondamentaux du travail comme acte politique de " confrontation du travailleur aux réalités de son milieu vital» (Supiot, 2017), elles portent aussi le risque d'éviter la conflictualité du travail et de mettre sous silence des enjeux sensibles entre travail, santé et société : les entreprises peuvent préférer parler de QVT plutôt que de RPS mais sans entreprendre le processus social et politique nécessaire à une QVT de qualité. Finalement, que l'on parle de démarche épidémiologique, de prévention des RPS ou de QVT, les mêmes questions se posent : comment amener les entreprises à considérer le psychosocial comme une ressource plutôt qu'un risque (Clot, 2010) ? Comment allier 
développement de la coopération entre les acteurs et développement de leur maturation autour du travail et de la santé (Nielsen \& Abildgaard, 2013) ? Comment permettre la révélation de l'activité (Cox et coll., 2010) et engager notamment les dirigeants supérieurs (Sarnin, 2016) ? Et surtout, comment passer du diagnostic à la transformation effective et durable des situations de travail et de l'organisation (Guidou, 2017 ; Debard, 2015) ?

La question n'est pas simple car le débat sur la santé constitue un objet polémique (Rouat et Sarnin, 2013). Par ailleurs, puisque les facteurs à l'origine des souffrances tiennent pour beaucoup aux difficultés des managers à piloter les organisations (Sarnin et coll., 2011), les préconisations issues des démarches remettent souvent en question les décisions prises dans le passé, ce qui équivaut à solliciter des acteurs pour «défaire» le travail accompli. Ajoutons que les acteurs doivent dialoguer à partir du travail réel alors même qu'une grande part de l'activité leur reste invisible (Davezies, 2012). Enfin, le rapport santé-travail construit par un individu au cours de sa carrière vient se jouer au sein du dialogue institutionnel sur la santé au travail et influence les comportements de santé (Sarnin et coll., ibid). Ainsi, si l'on souhaite que l'approche QVT se traduise par des pratiques efficientes, il semble pertinent de s'interroger sur l'ensemble des transformations exigées : que se passe-t-il quand l'intervention engage l'ensemble des acteurs de l'entreprise dans une telle démarche ? Cette question ouvre de nouvelles perspectives puisqu'il ne s'agit plus seulement de s'intéresser au cadre méthodologique nécessaire à une démarche QVT de qualité. Il s'agit plus fondamentalement de porter attention à la question des interactions existant entre le processus d'intervention et les acteurs de l'entreprise (Guidou, 2017).

Pour entreprendre cette réflexion, cet article présente une recherche-action (RA) réalisée dans une collectivité territoriale. Elle débuta par la commande d'une démarche de prévention des RPS comportant des demandes spécifiques. En tant que doctorante [nom du chercheur], nous avons proposé un dispositif permettant de répondre aux attentes formulées mais également à nos objectifs de recherche, c'est-à-dire la possibilité d'observer l'expérience vécue par les acteurs internes tout au long de l'intervention. Nous nous situons donc dans le cadre de la recherche-action (Rapoport, 1968/1973).

\section{Méthodologie}

\subsection{Problématique et cadre épistémologique}

Notre objectif est d'observer les interactions entre les acteurs de l'entreprise et le processus d'intervention d'une démarche d'amélioration des liens entre santé et travail, pour, à terme, mieux cerner les ressources et les freins rencontrés au cours de ces dispositifs. Notre démarche se rattache ainsi à une volonté de " travailler patiemment à cette " explication» [pour] se préparer à " comprendre » ce qui arrive en situation d'intervention [tout en] prenant le temps de s'affranchir du terrain, en transformant l'action de terrain en moyen de recherche »(Clot, 1995, p3). Cette RA s'inscrit donc dans le cadre de la recherche fondamentale de terrain (Clot, ibid).

Cette recherche exigeait d'expérimenter en situation réelle mais aussi de placer la démarche aux mains des acteurs internes afin d'être en mesure d'observer les interactions entre ces derniers et la démarche. La méthodologie a donc été construite pour que la démarche soit conduite par les acteurs internes et non pas pour ou même avec eux. Plusieurs points ont été 
contrôlés : s'assurer que les critères déontologiques sont respectés, vérifier que la démarche relève bien de santé au travail, choisir une méthodologie et s'y tenir, et y engager durablement un maximum d'acteurs internes. Dans notre cas, la commande formulée et le contexte d'intervention ont exigé le recours à une démarche par questionnaire. Malgré ses limites, cette méthodologie constitue toujours l'une des plus utilisées par les praticiens. En ce sens, étudier les interactions à l'œuvre entre les acteurs internes et son processus d'intervention, apparaît intéressant. Cette analyse complètera en effet les données scientifiques relatives à cette approche tout en commençant à collecter des données relatives aux expériences des acteurs internes.

\subsection{Contexte d'intervention}

La RA a été réalisée dans une collectivité territoriale, c'est-à-dire une entité administrative en charge des intérêts d'une population située sur un secteur géographique donné. Ses actions correspondent à plus de 100 métiers $^{1}$ exercés par plus de 3000 agents publics organisés autour d'un directeur général des services (DGS) et de directeurs généraux adjoints (DGA). La demande d'intervention était issue de la confrontation de la collectivité à trois types de risque. Le risque «psychosocial »d'abord: en plus d'une dégradation globale des indicateurs de santé dans les collèges ${ }^{2}$, deux agents avaient tenté de se suicider en quelques jours et un troisième s'était scarifié à son poste. Ces évènements ont entraîné un choc émotionnel, un questionnement des pratiques managériales ainsi qu'une crainte de suites médiatiques ou juridiques. Le risque «syndical » ensuite, le dialogue social étant particulièrement virulent. Le risque «politique » enfin : la collectivité constatait l'implantation croissante d'un autre parti ainsi qu'une prévalence des sentiments d'isolement et de défiance dans la construction de ce choix politique. À un an des élections, la majorité en place souhaitait donc agir pour (re)construire le sentiment de cohésion.

Dans ce contexte, la collectivité a souhaité une enquête de santé pour ses agents ${ }^{3}$ des collèges (plus de 600) exerçant dans plus de 60 établissements. La commande comportait trois aspects : inclure l'ensemble des agents, procéder par entretien individuel et réaliser une démarche incluant autant de représentants de l'administration (RAdm) que de représentants du personnel (RP). Au regard des enjeux, on comprend que ces demandes avaient pour objectif de (re)créer une proximité entre la collectivité et ses agents afin de renforcer les liens à l'employeur mais aussi à des fins stratégiques : les agents étant des citoyens appelés à voter prochainement, la collectivité espérait des retombées politiques. Au niveau interne, il s'agissait également de mener une action permettant d'obtenir l'adhésion des syndicats et donc d'améliorer le dialogue social. La genèse de l'intervention permet ainsi de souligner une réappropriation de la question psychosociale mais aussi une instrumentalisation à des fins stratégiques et politiques. Pour autant, elle renvoie également au premier mouvement d'intérêt stratégique des dirigeants pour la santé et représentait une opportunité pour accompagner le passage d'une vision en termes de « risques » à une vision en termes de « ressources ». Enfin, du côté de la recherche, cette demande offrait un moyen d'observer les interactions entre des acteurs internes fortement impliqués et le processus d'intervention.

\footnotetext{
${ }^{1}$ Les nombres sont volontairement très larges afin de préserver l'identité de la collectivité.

2 Etablissements scolaires français accueillant des élèves âgés de 11 à 15 ans environ.

${ }^{3}$ Chef de cuisine, agent de restauration, agent d'accueil, agent de maintenance, agent chef et agent d'entretien.
} 


\subsection{Dispositif méthodologique}

Pour répondre à nos objectifs de recherche, nous avons limité notre participation à la construction d'un dispositif assurant les conditions de contrôle présentées plus haut. Concernant le choix méthodologique, nous avons proposé de structurer les entretiens individuels par un questionnaire. Ce choix semblait en effet indispensable pour répondre aux questions soulevées par la commande, notamment l'organisation d'entretiens avec plus de 600 agents répartis dans plus de 60 lieux tout en espérant une analyse qualitative et synthétique. L'approche par questionnaire était intéressante car elle impose le cadre déontologique et freine les risques d'instrumentalisation : le questionnaire structure les entretiens à l'identique et oblige à vérifier un certain nombre de critères de confidentialité et de transparence ${ }^{4}$. Une fois ces conditions garanties, nous avons veillé à ne jamais introduire nous-mêmes d'axes de réflexion, limitant nos interventions aux seuls points soulevées par les acteurs internes au fil de leurs discussions pour construire et conduire la démarche.

La gouvernance du dispositif a été structurée à deux niveaux. Un comité de pilotage restreint a été créé. Il se composait de deux DGA, d'une chargée de mission auprès du DGS et de nous-mêmes en tant que doctorante [nom du chercheur]. Ce pré-travail était très rapidement présenté à un second niveau de pilotage transversal composé par 22 RAdm et 22 RP. Les premiers ont été choisis parmi les encadrants supérieurs et les seconds parmi les organisations syndicales selon une répartition proportionnelle à leur représentativité.

Tableau 1 : Caractéristiques de la population du collectif d'enquêteurs

Tous les éléments de l'enquête ont été mis en débat au sein du collectif RAdm-RP pour obtenir des compromis devenant constitutifs de la démarche : objectifs, conditions déontologiques, variables du questionnaire, formulation des items, cotation, questions d'amorce, modes de passation des entretiens, recueil des réponses, communication .... Il a ainsi été décidé que les agents seraient rencontrés par un duo composé d'un RAdm et d'un $\mathrm{RP}^{5}$ et que ces derniers seraient en charge de la co-rédaction des comptes-rendus $(\mathrm{CR})$ rédigés sur la base du questionnaire et à nous transmettre. Une charte éthique stipulait la confidentialité des entretiens et obligeait à la destruction des CR une fois transmis. Ces derniers ont ensuite été traités statistiquement par nos soins via le logiciel Spad. Enfin, les résultats ont été présentés au collectif d'enquêteurs sans y inclure de préconisations. Il s'agissait en effet de les replacer dans un rôle actif afin qu'ils co-produisent les transformations et que nous puissions poursuivre l'observation des interactions lors de ces dernières étapes.

\section{Résultats}

\subsection{Evolution des interactions entre acteurs internes et processus d'intervention}

\footnotetext{
${ }^{4}$ Déclarations règlementaires, identification de responsables, règles de respect de l'anonymat et de traitement des données recueillies, garantie des droits des répondants, ...

${ }^{5}$ Composition contrôlée par tirage au sort
} 
Pour conserver la clarté de notre propos, nous ne présenterons que les résultats en lien avec les interactions qui nous intéressent. Rappelons les caractéristiques de l'intervention : être entièrement remise aux mains d'acteurs internes tant dans ses phases de conception, de réalisation que de transformation; ces acteurs internes constituant un collectif composé d'autant de RP que de RAdm (choisis parmi les encadrants supérieurs) et enfin s'appuyer sur un questionnaire administré au cours d'entretiens de face à face co-conduits par des duo RAdm et RP. Le premier indicateur des interactions entre acteurs internes et processus d'intervention renvoie à l'ampleur de la démarche : durant 8 mois $^{6}$, les binômes RAdm-RP ont réalisé 515 entretiens auprès d'agents volontaires. 487 CR ont été co-rédigés, saisis et traités ${ }^{7}$. Au total, 2912 heures ont été consacrées à la démarche. Ces résultats, impressionnants, confirment l'importance d'une appropriation des enjeux de santé par l'employeur, notamment lorsqu'elle articule performances économiques ${ }^{8}$ et sociales. Ils soulignent également les effets d'une haute implication des acteurs internes, en termes de participation aux différentes étapes mais aussi de positionnement dans un rôle actif.

Pour compléter ces résultats avec des données qualitatives, nous avons relevé les interrogations soulevées durant les espaces de construction et de conduite de la démarche. Elles constituent en effet des indicateurs des interactions qui nous intéressent. Nous les avons ensuite catégorisées en thématiques.

Tableau 2 : Présentation des réunions du collectif d'enquêteurs et des questions soulevées

Ces résultats témoignent du nombre d'espaces nécessaires aux enquêteurs pour se saisir des principaux axes de la démarche : 12 réunions et $29 \mathrm{~h} 30$ de discussions ont en effet été réalisées. La catégorisation des interrogations permet également de cerner la complexité des connaissances à acquérir : durant les premières étapes, les thématiques renvoient aux savoirs entourant la dynamique santé-travail et à des compétences «techniques » nécessaires. En revanche, une fois les entretiens débutés, on note une évolution des thématiques vers une mise au travail des représentations sur les liens entre santé-travail.

\subsection{L'évolution des connaissances et des représentations}

$\mathrm{Au}$ fil de la démarche, nous avons observé une évolution des connaissances et des représentations des acteurs internes sur les liens santé-travail. Elle a été particulièrement visible durant les premières étapes de construction du questionnaire puisque les enquêteurs ont formulé d'eux-mêmes, de nombreuses interrogations. Aussi, pour leur répondre sans introduire nous-mêmes d'orientations, nous avons présenté deux exemples de questionnaires couramment utilisés ${ }^{9}$. Les questions techniques qu'ils ont continué de poser, notamment sur la pertinence des items, ont abouti à une diffusion des connaissances sur les liens entre santé et travail. Par exemple, les items concernant la qualité du travail ont permis de transformer les

\footnotetext{
${ }^{6}$ La durée de 8 mois renvoie uniquement aux temps nécessaires à la construction du dispositif et à la réalisation des entretiens, l'ensemble de la démarche s'est étalé sur une année.

${ }^{7}$ L'écart correspond aux comptes-rendus non remis ou remis trop tardivement pour être inclus dans l'analyse

${ }^{8}$ Il nous semble en effet que les performances électorales ou liées à la qualité du service public peuvent renvoyer aux performances économiques évoquées lorsque l'on parle du secteur privé.

${ }^{9}$ Questionnaires SUMER (file:///C:/Users/Nad\%C3\%A8ge/Downloads/ac88.pdf) et Evrest (http://evrest.istnf.fr)
} 
représentations des métiers, certains RAdm et RP considérant que le métier d'agent d'entretien "était dénué d'intérêt et qu'il serait donc mal venu d'interroger le sentiment de bien faire son travail ». Les questions relatives aux objectifs de la démarche ont permis des échanges autour de la définition de la santé, non comme une absence de difficultés mais comme la possibilité d'agir créativement sur elles. Ces discussions ont eu un effet réflexif. Par exemple : "De toute façon, c'est illusoire de vouloir que tout le monde s'entende bien dans le travail et puis, quand on n'est pas d'accord et qu'on peut en parler, ça peut aussi aboutir à de bonnes idées, n'est-ce pas $M P$ » (un RP qui s'adresse au DGA 1 avec humour). Ou encore "C'est sûr que j'ai rencontré des personnes qui pensent que mon travail est sans intérêt parce que je calcule des trucs toute la journée alors que pour moi, c'est intéressant! Il ne faudrait pas qu'on fasse la même chose avec les agents » (un RP exerçant une fonction comptable). "Finalement, ces questionnaires parlent quand même beaucoup plus du travail que des problèmes de santé des personnes!» (un directeur).

Le questionnaire élaboré par le collectif témoigne de ce travail de co-construction et rend compte de l'évolution des connaissances et des représentations des acteurs (voir annexe). Il comporte des questions pour chacun des facteurs RPS généralement interrogés par les outils scientifiques. De même, la cotation des réponses par des échelles (de type Likert) permet d'obtenir une finesse des avis et des données chiffrées. Certaines dimensions sont toutefois moins bien questionnées ou ne l'ont pas été (l'intensité qualitative, l'équilibre avec la vie privée, la part créative du travail et sa répétitivité). On note également que de nombreuses questions ont été ajoutées (soulignées dans le questionnaire en annexe), révélant ainsi une adaptation aux métiers et au contexte. Des thèmes liés aux objectifs stratégiques de la collectivité ont aussi été ajoutés (lien à l'employeur, éléments de ressources humaines). Enfin, les questions sensibles n'ont pas été supprimées, bien au contraire (recensement de 12 atteintes de la santé, jugement des agents sur le rôle du travail dans celles-ci, perception de la collectivité). Rappelons que ces interrogations exposaient fortement notamment du fait de la présence des RP. Finalement, l'autonomie des enquêteurs n'a donc pas eu d'impacts négatifs majeurs sur la qualité du questionnaire. L'implication des cadres supérieurs a abouti à l'intégration de questions stratégiques, sans aucune forme de censure. Ainsi, en considérant l'ensemble des interactions entre acteurs internes et processus d'intervention, il semble possible de dire que les membres du collectif sont passés d'une vision de l'enquête de santé comme un relevé des atteintes, à une recherche de facteurs en lien avec le travail, le management, l'organisation et l'institution; et ce, malgré les connaissances et compétences à acquérir ainsi que la perturbation de leurs représentations.

\subsection{Les difficultés liées au passage à l'action}

Malgré les espaces de coopération, la haute implication des acteurs internes, l'ampleur de la démarche ou encore l'évolution des connaissances et des représentations, les observations réalisées lors de l'élaboration des préconisations, ont permis d'identifier d'importantes difficultés. En effet, à l'issue de la présentation des résultats, RAdm comme RP n'ont pas été en mesure de se saisir de ces ressources : une seule préconisation a été émise, à savoir des concours de type «Top chef » pour les chefs de cuisine afin de montrer la richesse des savoirfaire. Nous avons identifié deux types de frein. Les premiers sont de nature cognitive et renvoient à la complexité à se saisir de l'ensemble des variables agissant sur la santé. $\mathrm{Ne}$ parvenant pas à appréhender la combinaison des influences, le collectif a finalement proposé un seul levier (s'appuyant sur les résultats des variables liées à la reconnaissance). Ces 
difficultés peuvent sans doute être attribuées à la nature épidémiologique du questionnaire : les résultats montrent les atteintes alors que les préconisations visent ce qui protège et développe la santé. De même, la santé est présentée comme morcelée (relevant de différentes variables) alors que l'élaboration des préconisations nécessite une articulation globale des facteurs. Pour autant, notons que ces carences 'techniques' avaient été surmontées par les acteurs lors des étapes de construction. Un second phénomène est en effet apparu. Durant la construction du questionnaire, le collectif avait posé de lui-même des questions techniques, nous amenant ainsi à donner des exemples d'outils permettant, in fine, un transfert méthodologique. Or, durant les étapes d'élaboration des préconisations, les questions techniques ont disparu pour laisser place à l'expression de surprises et à des verbalisations sur leurs propres vécus et rapport au travail ${ }^{10}$ :

Tableau 3 : exemples d'échanges lors des réunions consacrées aux préconisations

Nos observations montrent que les espaces de discussion consacrés au passage à l'action ont été envahis par des verbalisations liées à l'expérience vécue par les acteurs internes au cours de la démarche. Leur haute implication les a amenés à vivre une expérience personnelle, nous pourrions dire une expérience réflexive sur leur propre rapport au travail. Cette expérience s'est accompagnée d'une incapacité à inscrire ce débat dans le futur et dans un autre travail que le leur. Ces freins semblent donc cette fois de nature psychologique.

\section{Discussion}

Ces premiers résultats concernant l'interaction entre acteurs internes et processus d'intervention au cours d'une démarche de santé au travail demandent à être complétés et enrichis. L'obligation d'expérimenter en situation réelle et de placer la démarche aux mains des acteurs internes entrainent des limites méthodologiques même si cela constitue une forte originalité. Premièrement, le questionnaire construit - même s'il comprend la majorité des items présents dans ces outils - n'est pas un outil scientifiquement validé. Par ailleurs, les précautions prises pour standardiser la passation ne peuvent garantir une absence de biais. Aussi, même si les résultats de l'enquête ne constituaient notre principal objet de travail, nous pouvons considérer que les données à partir desquelles les acteurs ont réfléchi, sont critiquables. Précisons toutefois que les résultats du questionnaire étaient cohérents avec les données scientifiques. Une dernière limite relève de l'obligation de recourir à un questionnaire du fait des contraintes de la commande. Plusieurs travaux ont en effet déjà montré la complexité à se saisir des résultats statistiques induits par ces outils. Pour autant, nos observations montrent qu'au-delà de cette complexité disons mathématique, la difficulté est aussi de réfléchir aux préconisations censées protéger et développer la santé, à partir de données portant uniquement sur les altérations causées par le travail sur la santé. Or, nous avons rappelé en introduction que bien d'autres méthodologies restent centrées sur les altérations, y compris des approches qualitatives.

\footnotetext{
${ }^{10}$ Tenant notre posture d'observateur, nous n'avons donc pas (à ce stade) introduit d'éléments influant sur les interactions entre acteurs internes et processus d'intervention.
} 
Nos résultats confirment tout d'abord qu'une forte participation des acteurs internes développe leurs connaissances et représentations sur la dynamique santé-travail. Cette haute implication, entendue comme participation et posture actives, a permis de construire une démarche globalement de qualité intégrant des dimensions stratégiques et de performance. Elle a ainsi permis le passage d'une démarche d'évaluation des RPS à une démarche relevant davantage de la QVT. En revanche, lors du passage vers la transformation, des freins importants ont été observés. Ainsi, la haute implication des acteurs internes, l'intégration des managers supérieurs, tout comme l'ajout de dimensions stratégiques, semblent nécessaires à l'efficience d'une démarche mais non suffisants. Le rôle d'observateur que nous avons occupé, permet de renforcer ces résultats : en répondant uniquement aux interrogations soulevées par les acteurs, nous avons bien été en mesure d'observer l'évolution de ce collectif au fil de la démarche. Les observations offrent une première compréhension des freins existants. Les démarches qui se centrent sur les impacts négatifs du travail sur la santé engendrent des freins cognitifs. Elles amènent les acteurs à réfléchir à partir des altérations et les conduisent ainsi à imaginer des mesures permettant de les réduire. Le processus cognitif implanté relève ainsi d'une logique tertiaire et non primaire. Ces freins ont été confirmés durant les suites de la démarche lorsque, disposant alors de la possibilité de quitter la position d'observateur, nous avons introduit des préconisations. Le processus d'intervention s'est alors poursuivi pour aboutir à des changements. Ces résultats montrent donc la nécessité d'un soutien méthodologique et technique tout au long de la démarche mais sans doute encore davantage lors de l'élaboration des préconisations. C'est l'apparition de freins de nature psychologique qui explique, selon nous, cette complexité accrue. Ces résultats révèlent un aspect encore peu abordé dans la littérature : celui du vécu des acteurs au gré d'une démarche. En effet, beaucoup de travaux ont montré que les démarches ne pouvaient faire l'économie d'agir sur les connaissances et représentations des acteurs, les invitant ainsi à réinterroger le travail. Mais dire cela, c'est aussi dire que les interventions provoquent une perturbation des acteurs au sens de Devereux (1967). Le sujet possède en effet cette "capacité émergente d'accueillir du sens et d'en faire quelque chose pour soi»(Castoriadis, 1990). Ainsi «la coconstruction [n'est pas] à comprendre comme la participation des individus ou des groupes, à leur insu, à la production d'un matériau [...]. L'activité représentative, compréhensive, symbolisante des sujets est incluse dans l'objet de recherche » (De Gaulejac et coll., 2013, p22). Ainsi, nous pouvons considérer que l'absence de soutien dans cette perturbation, participe de la difficulté à passer à l'action transformatrice.

En conclusion, cette RA a permis d'observer plusieurs processus importants pour la recherche : tout d'abord, une haute implication des acteurs internes soutient le développement de démarches relevant de la QVT. Ensuite, ces acteurs rencontrent des freins cognitifs et psychologiques qui entravent l'intervention. Les premiers sont transversaux à l'ensemble de la démarche mais sont complétés par des freins psychologiques apparaissant lors du passage entre le diagnostic et la transformation. Nous comprenons ce résultat comme un phénomène de perturbation de ces acteurs, ou plutôt, de ces sujets. En ce sens, les démarches de prévention devraient nécessairement considérer l'ensemble des interactions entre les acteurs internes et le processus d'intervention, y compris dans leurs dimensions intersubjectives et intrasubjectives. Nos résultats montrent que ces interactions doivent être nombreuses et ne pas amener les acteurs à réfléchir uniquement à partir des altérations. Pour autant, puisque les interventions modifient le rapport des acteurs à leur travail, cet effet doit être pris en compte, que ce soit pour mieux comprendre les processus à l'œuvre, pour permettre une action 
transformatrice plus efficiente ou tout simplement - mais non moins fondamentalement d'un point de vue éthique. La question de l'interaction entre les acteurs et le processus d'intervention semble donc une voix pertinente pour les recherches à venir. 


\section{Bibliographie}

Althaus, V. (2013). Élaboration et mise en ouvre d'une démarche d'intervention systémique pour les PME : construction théorique et application pratique dans cinq entreprises (Thèse de doctorat, Université de Lorraine à Nancy, France). Récupéré de l'archive de publication électronique de l'Université de Lorraine: http://docnum.univlorraine.fr/public/DDOC_T_2013_0299_ALTHAUS.pdf.

Askenazy, P., Baudelot, C., Brochard, P., Brun, J.P., Cases, C., Davezies, ... et Weill-Fassina, A. (2010). Mesurer les facteurs psychosociaux de risque au travail pour les maîtriser: rapport du collège d'expertise sur le suivi des risques psychosociaux au travail, faisant suite à la demande du Ministre du travail, de l'emploi et de la santé. Paris. Récupéré le 06/01/2011.

emploi.gouv.fr/IMG/pdf/rapport_SRPST_definitif_rectifie_11_05_10.pdf http://travail-

Boussard, V. (2009). Les consultants au cœur des interdépendances de l'espace de la gestion. Cahiers internationaux de sociologie, 126, 99-113.

Caillé, A., Jeoffrion, C. (2017). Prévention des risques psychosociaux au sein d'établissements publics d'enseignement agricole: quand le diagnostic organisationnel participe d'une amélioration de la qualité de vie au travail. Psychologie du Travail et des Organisations, Volume 23, 4, 308-325.

Castoriadis, C. (1990). Les carrefours du labyrinthe, t.3, Le monde morcelé. Paris : Le Seuil

Clot, Y. (2010). Au-delà de l'hygiénisme du travail : l'activité délibérée. Nouvelle revue de psychosociologie, 10, 41-50.

Clot, Y. (1995). Le travail sans l'homme? Pour une psychologie des milieux de travail et de vie. Paris : La découverte.

Clot, Y., Davezies, P. (2011). Des accords sur le stress qui sonnent faux. Santé \& Travail, 74, 26-29.

Cox, T., Taris, T., Nielsen, K. (2010). Organizational interventions : issues and challenges. Work \& stress, 24, 3, 217-218.

Davezies, P. (2012). Enjeux, difficultés et modalités de l'expression sur le travail : point de vue de la clinique médicale du travail. PISTES, 14-2. https://pistes.revues.org/2566

Debard, A. (2015). La prise en compte des risques psychosociaux par les managers. Les processus soutenant l'action des encadrants en santé au travail (thèse de doctorat, Université Lumière Lyon 2, Lyon). http://theses.univ-lyon2.fr/documents/lyon2/2015/debard_a

Devereux, G. (1967). De l'angoisse à la méthode dans les sciences du comportement. Paris : Flammarion.

Fielding, N., Schreier, B.I. (2001). Introduction : In the compatibility between qualitative and quantitative research methods - Art.4. Forum : Qualitative research, 2(1).

Gaulejac, V. de., Giust-Desprairies, F., Massa, A. (2013). La Recherche clinique en sciences sociales. Paris : Erès. 
Grosjean, V., Guyot, S. (2016). Bien-être et qualité de vie au travail, risques psychosociaux : de quoi parle-t-on? Hygiène et Sécurité Du Travail, 242.

Guidou, N. (2017). La qualité, l'innovation et la créativité du travail au sein des organisations : vers une politique globale de prévention de la souffrance psychique au travail (thèse de doctorat, Université Lumière Lyon 2, Lyon). Récupéré de l'archive de publications électroniques de l'Université Lumière Lyon 2: http://theses.univlyon2.fr/documents/lyon2/2017/guidou_n

Jeoffrion, C., Hamard, J.P., Barré, S., Boudoukha, A.H. (2014). Diagnostic organisationnel et prévention des risques psychosociaux au sein d'un établissement d'accueil pour personnes âgées : l'intérêt d'une méthodologie mixte et participative. Le Travail Humain, 77(4), 373399.

Karasek, R. A. (1979). Job Demands, Job Decision Latitude and Mental Strain: Implications for Job Redesign. Administrative Science Quarterly, 24, 285-308.

Lachmann, H., Larose, C., Pénicaud, M. (2010). Bien-être et efficacité au travail. 10 propositions pour améliorer la santé psychologique au travail. Rapport pour le Premier ministre. Récupéré le 06/09/2012 :

http://www.ladocumentationfrancaise.fr/var/storage/rapports-publics/104000081.pdf

Lassare, D. (2005). Vers un modèle psychosocial de l'épisode de stress. In Chasseigne, G. et Lassarre, D. (dir.), Stress et Société, vol. 2 (p. 17-40), Reims : Presses universitaires de Reims.

Lazarus, R. S., Folkman, S. (1984). Stress, Appraisal, and Coping. New York: Springer Publishing Company.

Levet, P. (2013). Des risques psychosociaux à la qualité de vie au travail. Équiper les acteurs pour négocier le travail, l'expérience de l'ANACT. Négociations, 19, 97-111.

Ministère du travail, de l'emploi, de la formation professionnelle et du dialogue social. (2014). Arrêté du 15 avril 2014 portant extension d'un accord national interprofessionnel vers une politique d'amélioration de la qualité de vie au travail et de l'égalité professionnelle.

Récupéré le

03/06/2014 :

http://www.legifrance.gouv.fr/affichTexte.do?cidTexte=JORFTEXT000028867633

Nielsen, K., Abildgaard, G.S. (2013). Organizational interventions : A research-based framework for the evaluation of both process and effects. Work and stress, 27, 278-297.

Peters, S., Faulx, D., Hansez, I. (2010). Le rôle des objets-frontière dans le découpage temporel et social d'une innovation de service. Étude de cas d'un transfert de technologie depuis un laboratoire universitaire de sciences sociales. Revue d'Anthropologie des connaissances, 4, 65-86.

Ponnelle, S., Vaxevanoglou. X. (2017). Eléments de réflexion pour la mise en œuvre d'une démarche de prévention des risques psychosociaux au travail en France. Psychologie du Travail et des Organisations, Volume 23, 278-291.

Rapoport, R.N. (1973). Three dilemnas in action-research, Human Relations. Connexions, 7, 115-131. 
Rouat, S. (2010). Les processus individuels et organisationnels de construction de la santé au travail : prévention de la santé mentale au travail et analyse de dispositifs organisationnels Thèse de doctorat de l'université Lumière Lyon 2. http://theses.univlyon2.fr/documents/lyon2/2010/rouat_s

Rouat, S., Sarnin, P. (2013). Prévention des risques psychosociaux au travail et dynamique de maturation: le processus d'intervention comme opérateur de la transformation et du développement de la coopération. Activités, 10(1), 58-72.

Sarnin, P. (2016 juillet). L'engagement des dirigeants : enjeux et pistes d'action. Communication présentée au 19ème Congrès de l'Association Internationale de Psychologie du Travail de la Langue Française, Bruxelles.

Sarnin, P., Caroly, S., Douillet, P. (2011). Contre les « risques psychosociaux » : quels débats sur l'activité ? Le Travail Humain, 74(4), 309-320.

Siegrist, J. (2001). A Theory of Occupational Stress. In Dunham, W. (Eds.), Stress in the Workplace (p.55-66). London and Philadelphia : Whurr Publishers.

Supiot, A. (2017). Pour une réforme digne de ce nom : et si l'on refondait le droit du travail... Le Monde Diplomatique, 22-23.

Valléry, G. et Leduc, S. (2012). Les Risques Psychosociaux. Paris : PUF.

Vézina, M., Chenard, C., Chauvet, A., Jauvert, M. et Blanzina, I. (2015). Au-delà du risque psychosocial (RPS), quelle prise en compte des ressources professionnelles ? Dans Sarnin, P, Kouabenan, D.G., Bobilier Chaumon, M.E., Dubois, M \& Vacherand-Revel, J. (dir.), Santé et bien-être au travail (p.279-288). Paris : L'Harmattan.

Conflits d'intérêts : aucun 


\begin{abstract}
Annexe
Diagnostic global sur les conditions, l'environnement et le climat de travail Trame des entretiens semi-directifs - Visite des collèges
\end{abstract}

\title{
Les conditions de réalisation du travail
}

1. Pouvez-vous nous décrire les tâches que vous réalisez chaque jour ?

2. Pensez-vous que vous disposez du temps nécessaire pour faire correctement votre travail ?

OPas du tout d'accord OPas d'accord OD'accord OTout à fait d'accord Pouvez-vous nous expliquer? / Nous citer quelques exemples / Vous arrive-t-il d'être interrompu dans la tâche?

3. Pouvez-vous vous organiser comme vous le souhaiter?

OPas du tout d'accord OPas d'accord OD'accord OTout à fait d'accord

4. La plupart du temps, vous pouvez faire du travail de qualité.

OPas du tout d'accord OPas d'accord OD'accord OTout à fait d'accord

5. Globalement, estimez-vous que votre charge de travail a augmenté ?

OPas du tout d'accord OPas d'accord OD'accord OTout à fait d'accord Pouvez-vous nous citer des exemples?

6. Avez-vous la possibilité de prendre des initiatives ? De proposer des idées ?

OPas du tout d'accord OPas d'accord OD'accord OTout à fait d'accord

7. Dans votre travail, vous pouvez apprendre des choses nouvelles.

OPas du tout d'accord OPas d'accord OD'accord OTout à fait d'accord

8. Globalement, êtes-vous satisfait(e) de vos conditions de travail ?

OPas du tout OPlutôt non OPlutôt oui OTout à fait

9. Quelle est votre principale difficulté dans votre travail ?

10. Quelle est votre principale source de satisfaction dans votre travail ?

11. Disposez-vous des équipements suivants ?

O Un vestiaire suffisamment grand

O Une blouse de travail

Une douche

Des chaussures de travail

O Une salle de repos

Des gants

Un séchoir pour vos vêtements mouillés

O Autre vêtement de travail : ...

\section{L'organisation du travail}

12. La plupart du temps, votre travail vous semble-t-il bien organisé ?

OPas du tout d'accord OPas d'accord OD'accord OTout à fait d'accord 
13. Les missions et consignes qui vous sont données, sont claire.

OPas du tout d'accord OPas d'accord OD'accord OTout à fait d'accord

14. Recevez-vous des ordres contradictoires / opposés?

(Par exemple, respecter la règle : nettoyage des salles tous les jours à sec et un jour balayage

OJamais ORarement OLa plupart du temps OTout le temps

15. Votre (vos) responsable(s) est clairement identifié(s)

OPas du tout d'accord OPas d'accord OD'accord OTout à fait d'accord

16. Disposez-vous du matériel nécessaire à la réalisation de votre travail ?

OPas du tout d'accord OPas d'accord OD'accord OTout à fait d'accord

Facteurs liés aux relations de travail

17. Dans votre travail, vous êtes reconnu(e) par votre responsable

OPas du tout d'accord OPas d'accord OD'accord OTout à fait d'accord

18. En cas de difficultés, vous pouvez compter sur le soutien de votre responsable

OPas du tout d'accord OPas d'accord OD'accord OTout à fait d'accord

19. En cas de difficultés, vous pouvez compter sur le soutien de vos collègues

OPas du tout d'accord OPas d'accord OD'accord OTout à fait d'accord

20. Dans votre travail, les conflits sont réguliers

OPas du tout d'accord OPas d'accord OD'accord OTout à fait d'accord

21. Dans votre travail, il y a des moments où vous vous retrouvez tous en équipe de façon amicale

OJamais ORarement OLa plupart du temps OTous les jours

22. Vous rencontrez des difficultés avec les élèves et le personnel éducatif

OJamais ORarement OLa plupart du temps OTous les jours

23. Dans votre travail, il y a des injustices

OPas du tout d'accord OPas d'accord OD'accord OTout à fait d'accord

24. Dans votre travail, il existe des problèmes de communication

OPas du tout d'accord OPas d'accord OD'accord OTout à fait d'accord

\section{Travail et santé}

25. Sur une échelle de 1 à 5, comment jugez-vous votre état de santé ?

(1 très mauvais à 5 très bon)

$$
\mathrm{O} 1 \mathrm{O}_{2} \mathrm{O} 3 \mathrm{O} 4 \mathrm{O} 5
$$

26. Pensez-vous que votre travail a un impact sur votre santé ?

OPas du tout d'accord OPas d'accord OD'accord OTout à fait d'accord 
Quel est cet impact?

27. Vous disposez de tous vos équipements de protection individuelle (EPI)

OPas du tout d'accord OPas d'accord OD'accord OTout à fait d'accord

28. Concernant le renouvellement de vos EPI, comment cela s'organise-t-il ?

OChangement dès que l'EPI est usager

OChangement à chaque rentrée

OChangement tous les deux ans ou plus

29. Vous disposez de toute l'information nécessaire concernant l'utilisation des produits chimiques :

OPas du tout d'accord OPas d'accord OD'accord OTout à fait d'accord

30. Vous disposez de conseils ergonomiques sur votre poste de travail

OPas du tout d'accord OPas d'accord OD'accord OTout à fait d'accord

31. Avez-vous accès au registre hygiène et sécurité ?

\section{OOui ONon}

32. Au cours de l'année, avez-vous souffert des symptômes suivants sur une période de plus d'un $\underline{\text { mois }}$

Troubles du sommeil (réveils, insomnie, cauchemar, ...) $\quad$ Hypertension

Troubles de l'appétit

O Irritabilité

O Douleurs dorsales

Difficulté de concentration

Troubles digestifs

Troubles de la mémoire

O Stress

O Grande fatigue

Angoisse sur le chemin du travail

Etat moral dépressif

33. D’une façon générale, pour vous, votre travail est source de

B Bien-être $O$ Souffrance $\mathrm{O}$ 'a aucun impact sur moi

\section{$\underline{\text { Liens avec l'employeur }}$}

34. Vous connaissez bien l'organisation du ....

OPas du tout d'accord OPas d'accord OD'accord OTout à fait d'accord

35. Avez-vous un accès facile à un ordinateur

\section{OOui ONon}

36. Vous savez à qui vous adresser si vous recherchez une information

OPas du tout d'accord OPas d'accord OD'accord OTout à fait d'accord

37. Dans votre quotidien, le ... représente

Une aide, un soutien 
L La structure qui verse mon salaire

Une structure compliquée

Une administration qui ne porte pas attention à moi

O Rien

38. Vous disposez de suffisamment d'informations sur

Le suivi de votre carrière

La rémunération

O La formation

L Les conditions de travail

O Le service de la Direction de ....

39. Qu'attendez-vous précisément, de plus, de la part de votre employeur ...

40. Avez-vous des questions ou d'autres éléments à nous transmettre et qui n'auraient pas été abordés dans cet entretien?

41. A ce jour, souhaitez-vous changer de travail ou de métier ?

OOui ONon

Si oui, qu'aimeriez-vous faire?

Renseignements sur l'agent

Nom et prénom

Sexe : OOui ONon

Age

Situation familiale : OEn couple OSeul

Lieu de travail

Enfant à charge

Ancienneté dans le poste

Date de la dernière visite médicale 